\title{
Severe interstitial lung disease in connective tissue disease: rituximab
} as rescue therapy

\author{
Gregory J. Keir*, Toby M. Maher*, David M. Hansell", Christopher P. Denton`, \\ Voon H. Ong ", Suveer Singh ${ }^{+}$, Athol U. Wells* and Elisabetta A. Renzoni*
}

ABSTRACT: In very severe interstitial lung disease associated with connective tissue disease (CTD-ILD), progressing despite maximal conventional immunosuppression, there is no effective medical rescue therapy.

The aim of the present study was to test whether rituximab, a monoclonal antibody that depletes peripheral B lymphocytes, is effective as rescue therapy in very severe CTD-ILD, unresponsive to conventional immunosuppression.

We performed a retrospective assessment of eight patients with severe and progressive CTDILD treated with rituximab. In six patients, change in pulmonary function tests (PFTs) compared with pre-rituximab levels, was assessed at 9-12 months post-treatment. In two patients, who were mechanically ventilated at the time of treatment, clinical and HRCT changes were assessed.

Seven out of eight patients had a favourable treatment response to rituximab, while in one patient disease severity did not change. In contrast with previous progression, we observed a median significant improvement of $22 \%$ in diffusing capacity for carbon monoxide (from a median baseline of $25 \%$; range $16-32 \% ; p=0.04$ ), and a median significant improvement of $18 \%$ in forced vital capacity (from a median baseline of $45 \%$; range $37-59 \%$; $=0.03$ ), in the $9-12$ months following treatment with rituximab.

In very severe CTD-ILD unresponsive to conventional immunosuppression, rituximab may represent an effective, potentially life-saving, therapeutic intervention.

KEYWORDS: Collagen vascular disease, interstitial lung diseases

$\mathrm{n}$ the context of connective tissue disease (CTD), very severe interstitial lung disease (ILD) unresponsive to traditional treatment is an all-too-frequent and dispiriting experience. In the absence of lung transplantation, there is little to offer patients other than symptom palliation. In the idiopathic interstitial pneumonias, it has been observed that a palliative approach is appropriate once disease severity has reached a critical tipping point: despite a much lower mortality in earlier disease, idiopathic fibrotic nonspecific interstitial pneumonia (NSIP) has the same poor treated outcome as idiopathic pulmonary fibrosis (IPF) in patients with a diffusing capacity of the lung for carbon monoxide $(D \mathrm{~L}, \mathrm{CO})<35 \%$ predicted [1]. Clinical experience indicates that in the subgroup of patients with very severe, progressive CTD-ILD, prognosis is similarly bleak.
The pathogenesis of CTD-ILD is complex, and it is broadly accepted that underlying immune system dysfunction and immune-mediated pulmonary inflammation are critical to CTD-ILD development and progression. Abnormalities of cellular and humoral immune function have been described in ILD associated with systemic sclerosis (SSc) [2-4], idiopathic inflammatory myopathy $[5,6]$ and several other CTDs $[7,8]$. The success of antiinflammatory and immunosuppressive therapy in these conditions supports the notion that immunological over-activity is a key process in progressive lung fibrosis.

Rituximab is a chimeric (human/mouse) monoclonal antibody with a high affinity for the CD20 surface antigen expressed on pre-B and B-lymphocytes. Rituximab results in rapid depletion of $\mathrm{B}$ cells from the peripheral circulation [9], before B

\section{AFFILIATIONS}

*Interstitial Lung Disease Unit, Royal Brompton Hospital,

\#Dept of Radiology, Royal Brompton Hospital,

"Centre for Rheumatology and Connective Tissue Diseases, UCL Medical School, Royal Free Hospital, and

+Dept of Respiratory Medicine, Chelsea and Westminster Hospital, Fulham Road, Chelsea, London, UK.

\section{CORRESPONDENCE}

G.J. Keir

Interstitial Lung Disease Unit

Royal Brompton Hospital.

Sydney Street

SW3 6NP

London

UK

E-mail: G.keir@rbht.nhs.uk

Received:

Sept 202011

Accepted after revision:

Jan 022012

First published online:

Jan 262012 


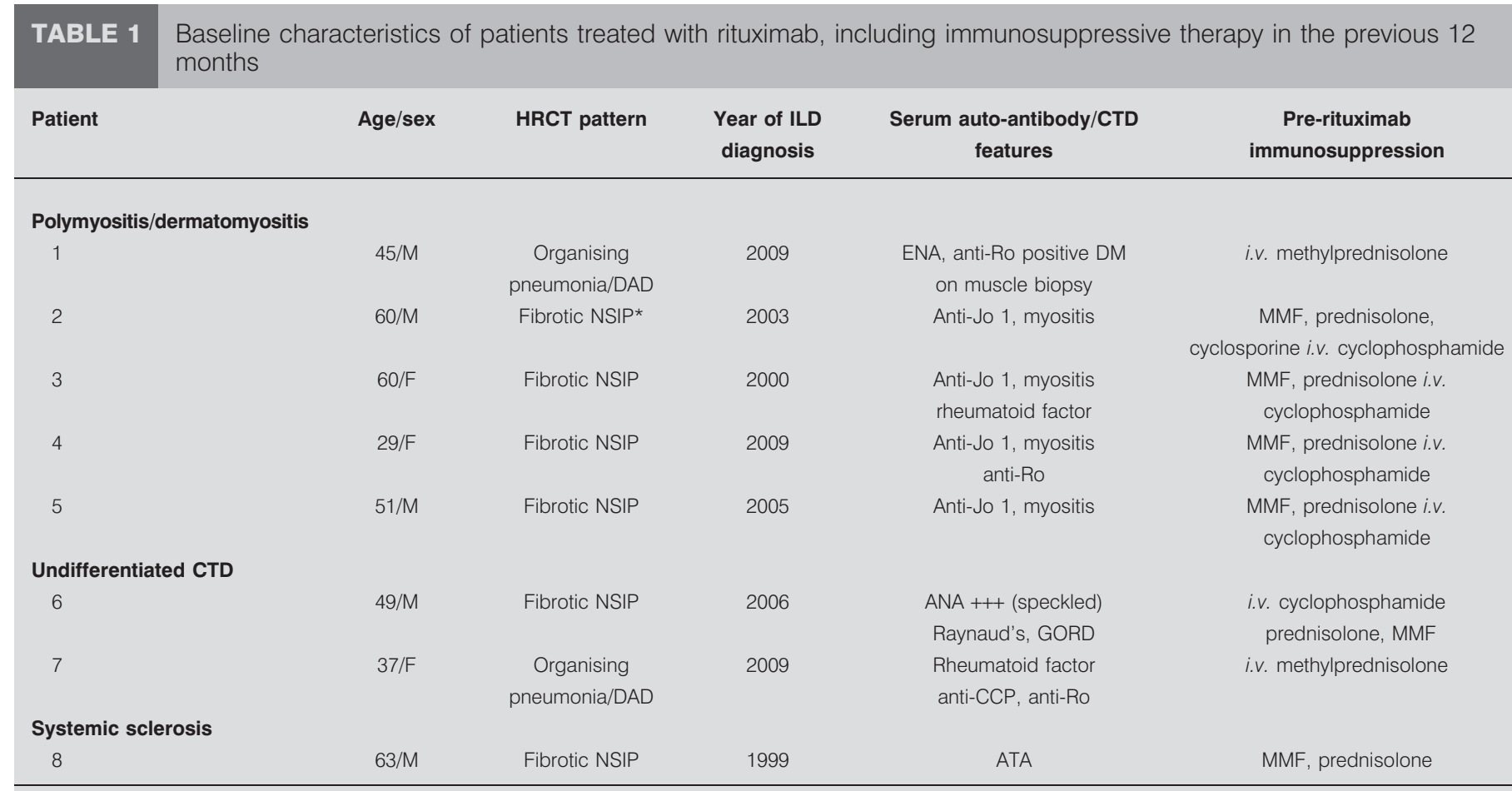

HRCT: high-resolution computed tomography; ILD: interstitial lung disease; CTD: connective tissue disease; M: male; F: female; DAD: diffuse alveolar damage; ENA: extractable nuclear antigen; DM: dermatomyositis; NSIP: non-specific interstitial pneumonia; MMF: mycophenolate mofetil; ANA: anti-nuclear antibody; GORD: gastrooesophageal reflux disease; CCP: cyclic citrullinated peptide; ATA: anti-topoisomerase antibody. *: fibrotic NSIP confirmed on surgical lung biopsy.

cell reconstitution begins 6-9 months later [10]. Evidence for the effectiveness of B cell depletion exists in a number of immunemediated conditions, including rheumatoid arthritis [11-13], anti-neutrophil cytoplasmic antibody-associated vasculitis [14, 15], pulmonary alveolar proteinosis [16] and immune thrombocytopenic purpura [17]. A few case series have suggest rituximab may also be effective in ILD occurring in the context of immunological over-activity, with favourable responses reported in anti-synthetase-associated ILD [18] and SSc-ILD [19]. However, patients in these series had interstitial lung involvement of predominantly mild-to-moderate severity.

Evidence for the effectiveness of rituximab as "rescue" therapy in patients with severe, life-threatening ILD is limited [20, 21]. We report the use of rituximab as rescue therapy in eight patients with underlying features of CTD, and exceedingly severe fibrotic lung disease, progressing in spite of vigorous conventional immunosuppression.

\section{METHODS}

\section{Patient groups}

Review of our medical records and hospital pharmacy prescribing database identified all patients treated with rituximab between December 2007 and December 2010. All consecutive patients with severe, progressive CTD-ILD were included in our analysis, with a total of eight patients. No patients with CTDILD were excluded due to poor outcome or early death. A minimum of 9 months post treatment follow-up was available for all patients. ILD occurred in association with polymyositis or dermatomyositis (PM/DM) in five patients, of whom four had anti-synthetase Jo-1 antibody. In two patients, ILD occurred in association with undifferentiated CTD, and in one with SSc. All patients had failed to respond to conventional immunosuppressive therapy, with ongoing deterioration in pulmonary function tests (PFTs) and/or worsening respiratory failure. At the time of referral, two patients were mechanically ventilated and transferred directly to the intensive care unit (ICU) of our hospital. Table 1 lists demographic information and immunosuppressive therapy in the 9-12 months prior to rituximab therapy.

\section{Statistical analysis}

All patients had PFT follow up of at least 9 months following rituximab. In the six patients with PFT data prior to rituximab treatment, the significance of median DL,CO and forced vital capacity (FVC) percentage change before and after treatment was assessed by Wilcoxon signed rank test. To evaluate statistical significance of response to treatment in all patients, categorical variables of change (worse, stable, improved) were generated by combining significant changes in pulmonary function tests (defined as a change in FVC of $\geqslant 10 \%$ and/or a change in $D \mathrm{~L}, \mathrm{CO}$ of $\geqslant 15 \%$ ) and/or clinical status (for the two patients requiring mechanical ventilation, the requirement for mechanical ventilation was classified as a significant deterioration). Change was assessed 9-12 months before and after rituximab, and analysed using the Wilcoxon signed-rank test. A p-values of $\geqslant 0.05$ was considered statistically significant. Analysis of follow-up data at 9-12 months was chosen based upon previously reported responses of CTD-ILD to rituximab $[18,19]$, and the expected duration of B cell ablation following rituximab $[10,15]$. 

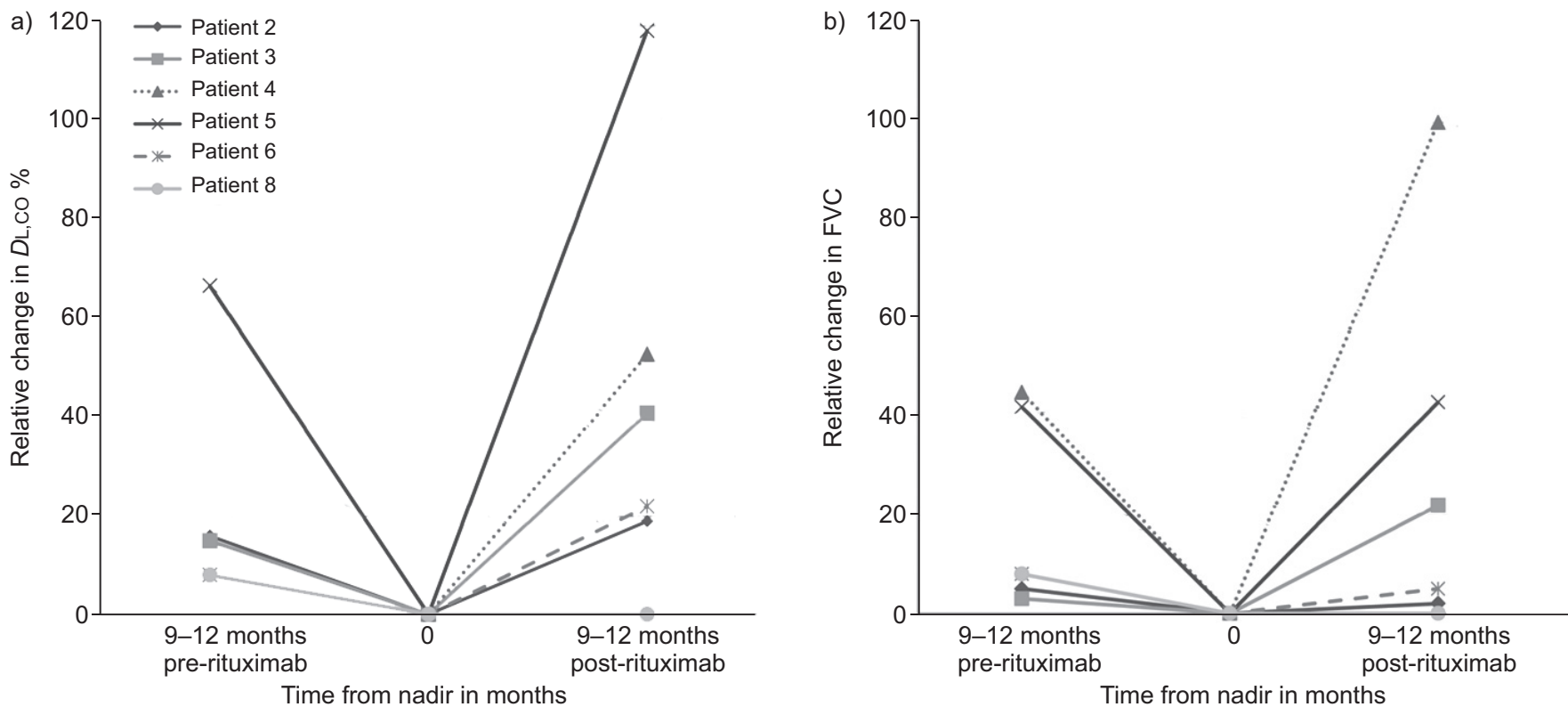

FIGURE 1. a) Change in diffusion capacity of the lung for carbon monoxide $(D L, C O) \%$ predicted in the $9-12$ months before and after rituximab administration. Following rituximab, there was a median improvement in $D \mathrm{~L}, \mathrm{CO} \%$ pred of $22 \%$ (range $0-119 \%$; $\mathrm{p}=0.04$ ), in contrast to a median decline of $16 \%$ (range $8-67 \%$ ) prior to rituximab. b) Change in forced vital capacity (FVC) \% pred in the 9-12 months before and after rituximab administration. Following rituximab, there was a median improvement in FVC \% pred of $18 \%$ (range $0-100 \% ; p=0.03$ ), in contrast to a median decline of $29 \%$ (range $3-45 \%$ ) prior to rituximab.

\section{Patient assessment}

All patients were discussed at our interstitial lung disease multidisciplinary meeting and underwent a full clinical assessment, including high-resolution computed tomography (HRCT) and PFTs.

\section{Rituximab treatment}

Rituximab was administered in accordance with our hospital New Drugs and Clinical Guidelines Group recommendations. The treatment protocol consists of rituximab 1,000 mg administered on day 0 and day 14 (preceded by treatment with intravenous hydrocortisone and chlorphenamine), the dosing regimen approved for rheumatoid arthritis treatment [12]. Patient 5 received rituximab $375 \mathrm{mg} \cdot \mathrm{m}^{-2}$ weekly for 4 weeks, administered before our hospital guidelines were finalised. Following rituximab administration, requirements for ongoing immunosuppression were assessed on an individual patient basis.

\section{RESULTS}

At the time of rituximab treatment, all patients had extremely severe ILD, with a median DL,CO of $25 \%$ (range 16-32\%) and FVC of $45 \%$ (range $37-59 \%$ ), and an anticipated survival of less than 6-12 months (based on clinical experience and survival data in patients with idiopathic fibrotic ILD of similar severity) [1]. Following rituximab, we observed a significant categorical improvement in clinical status and/or PFTs (DL,CO and/or FVC) in seven out of eight patients $(p=0.008)$ according to the criteria outlined in the methods section. In one patient (patient 8), disease severity did not appear to change following treatment, other than mild symptomatic improvement. In six patients with serial PFTs, there was a median improvement in DL,CO of $22 \%$ (range $0-119 \%$; $p=0.04$ ) and FVC of $18 \%$ (range $0-100 \%$; $\mathrm{p}=0.03$ ) within $9-12$ months of rituximab administration. This improvement occurred following a median decline in $\mathrm{DL}, \mathrm{CO}$ of $16 \%$ (range $8-67 \%$ ) and in FVC of $29 \%$ (range 3-45\%) in the 9-12 months preceding rituximab treatment (fig. $1 \mathrm{a}$ and $\mathrm{b}$ ). Improvements in PFTs and/or clinical status tended to occur rapidly (within 2-3 months in most patients), and were also statistically significant $(\mathrm{p}<0.05)$ when analysed at the earlier time point of $6 \pm 1$ months post-rituximab treatment.

Five patients had a striking response to rituximab, with dramatic improvements in lung function (patients 3,4 and 5) and/or successful extubation (patients 1 and 7). Change in clinical status and PFTs are summarised in table 2. Supplementary figure S1a-f shows lung function responses to treatment in individual patients. Additional clinical information can be found in the online supplementary material, including complete functional follow up.

\section{Polymyositis/dermatomyositis-associated ILD}

\section{Patient 1}

This 45-yr-old male was transferred to the ICU at our hospital after failed extubation following a quadriceps muscle biopsy procedure. Recently diagnosed dermatomyositis with associated organising pneumonia had deteriorated despite i.v. methylprednisolone and, at transfer, HRCT demonstrated a pattern of organising pneumonia with admixed fibrosis and diffuse alveolar damage (fig. 2a). The combination of lifethreatening respiratory failure, HRCT findings and the underlying autoimmune process resulted in the decision to treat concurrently with i.v. cyclophosphamide $750 \mathrm{mg}$ and rituximab. A dramatic improvement in HRCT appearances occurred over the following six weeks (fig. $2 \mathrm{a}$ and $\mathrm{b}$ ), and improvements in 


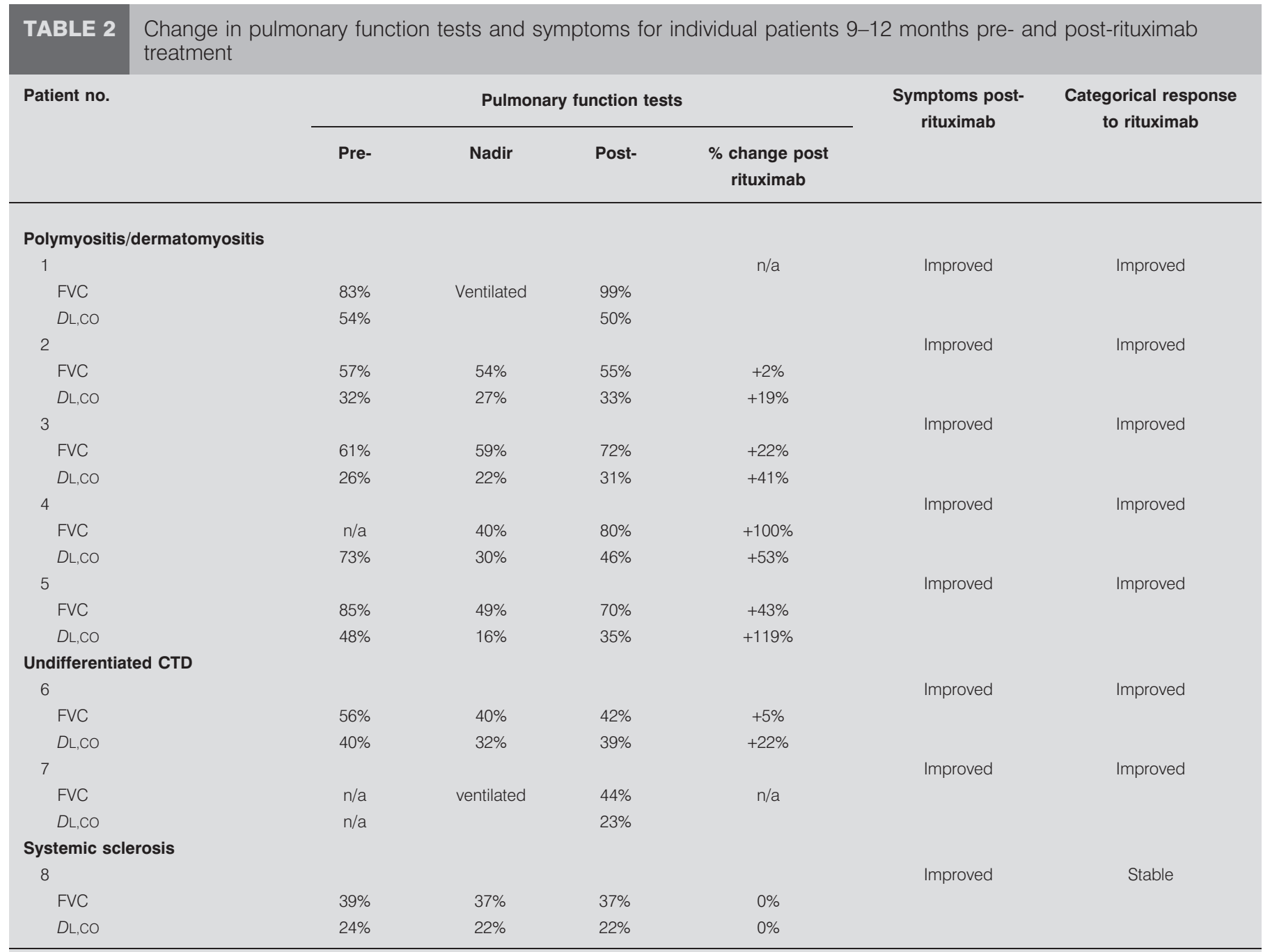

Nadir values represent the pulmonary function test measurements (or requirement for mechanical ventilation), immediately prior to rituximab administration. FVC: forced vital capacity; DL,CO: diffusing capacity of the lung for carbon monoxide; CTD: connective tissue disease.

PFTs have continued during follow-up (with a DL,CO of 50\% and FVC 99\% predicted at most recent review).

\section{Patient 2}

This 60-yr-old male with longstanding PM (Jo-1 antibody positive)-associated fibrotic NSIP, experienced gradual respiratory deterioration and persisting myositis activity despite concurrent mycophenylate, cyclosporine and prednisolone. Within 9 months of rituximab, serum creatine kinase had decreased from 4,500 to $370 \mathrm{IU} \cdot \mathrm{L}^{-1}$, and there was a significant subjective improvement in muscle strength and dyspnoea, with a $19 \%$ improvement in DL,CO (table 2).

\section{Patient 3}

This 60-yr-old female with anti-synthetase antibody (Jo-1)-associated fibrotic NSIP experienced a 12-month history of worsening symptoms and deteriorating PFTs despite i.v. cyclophosphamide. Following rituximab, there was a striking improvement in respiratory and extra-pulmonary symptoms within 3 months, coupled with improvements in PFTs (table 2).

\section{Patient 4}

This 29-yr-old female was referred with rapidly progressive fibrotic NSIP associated with anti-synthetase syndrome (Jo-1), and deterioration despite prednisolone and mycophenylate, followed by i.v. cyclophosphamide. Rituximab resulted in major improvements in symptoms and PFTs within 2-3 months of administration, with improvements continuing over the subsequent 12 months of follow-up.

\section{Patient 5}

This 51-yr-old male was referred with very rapidly progressive fibrotic NSIP, associated with anti-synthetase syndrome (Jo-1), and deteriorating interstitial lung involvement despite prednisolone, mycophenylate and ultimately i.v. cyclophosphamide. Treatment with rituximab resulted in rapid and striking improvements in symptoms and PFTs (supplementary figure S1d), however he died in his local hospital 18 months following rituximab therapy (with the cause of death reported as a lower respiratory tract infection). During follow-up at our hospital 8 days before death, he remained well with stable 

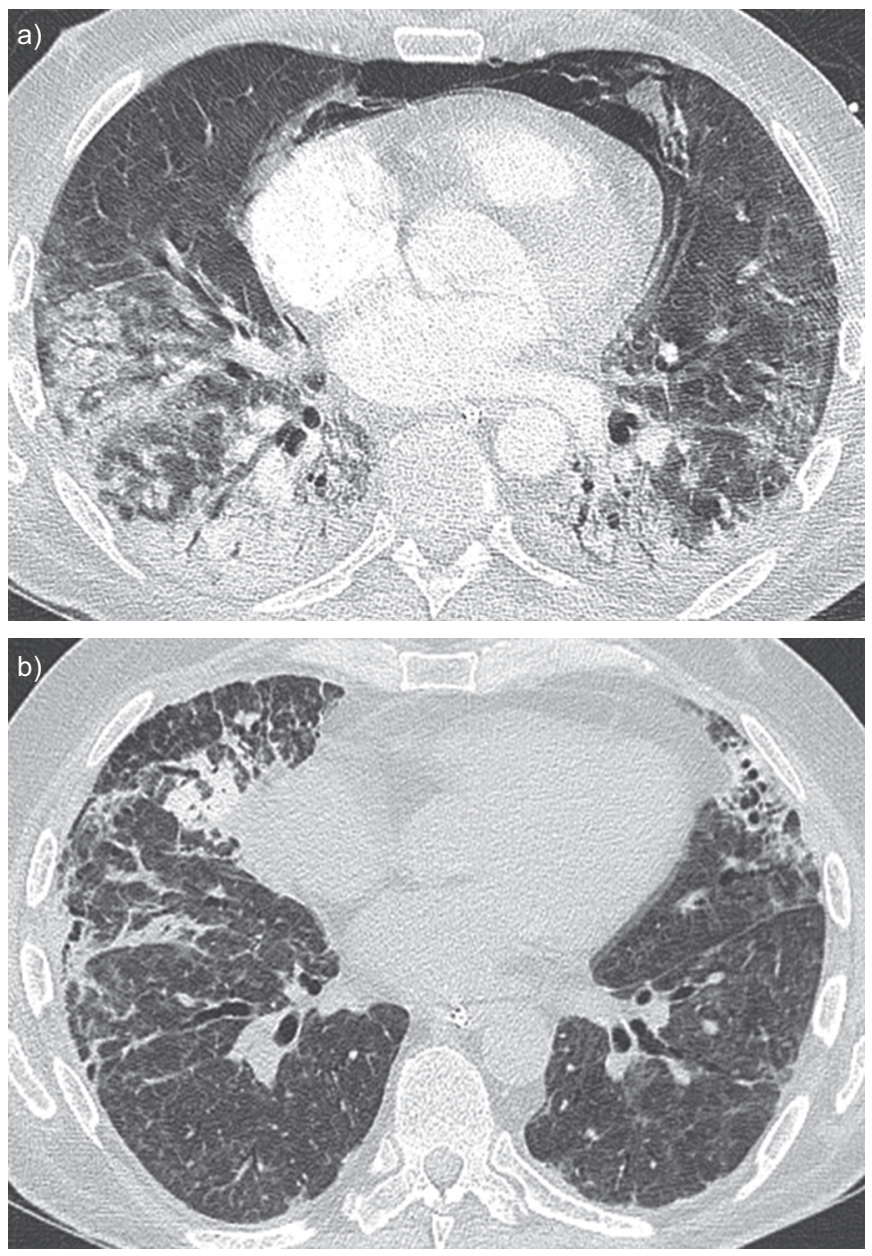

FIGURE 2. Patient 1. A 45-yr-old male with dermatomyositis. a) Contrastenhanced thin section CT shows patchy consolidation and ground glass opacification in the lower lobes, consistent with organising pneumonia and diffuse alveolar damage. A pneumomediastinum is present. b) 7 weeks following commencement of rituximab. The lower lobe consolidation and ground glass changes have almost completely resolved. Some of the reticular changes anteriorly, in the nondependent lung, were thought to be attributable to ventilator-induced damage.

PFTs (DL,CO of $38 \%$ and FVC of $68 \%$ ) and normal inflammatory markers. The most recent lymphocyte subset analysis performed 12 months following rituximab (and 6 months prior to death) revealed persisting B cell depletion although infections had not been problematic over this time. Cyclosporine had recently been commenced (in addition to a long-standing mycophenolate and prednisolone regimen), due to persisting myositis activity, and to allow further steroid reduction.

\section{Undifferentiated CTD}

Two patients in our cohort had a confirmed CTD with positive autoimmune serology and systemic symptoms (table 1), but did not fulfil the classification criteria for a defined CTD. Their clinical history is as follows.

\section{Patient 6}

This 49-yr-old male was diagnosed with an undifferentiated CTD in 2006, with HRCT appearances of fibrotic NSIP. Interstitial lung disease progressed despite i.v. cyclophosphamide, followed by mycophenolate and prednisolone. Rituximab was administered in December 2007, with a marked improvement in symptoms and lung function ( $22 \%$ improvement in $\mathrm{DL}, \mathrm{CO})$ over the next 9 months, before deterioration recurred (despite continued oral immunosuppression). He was declined for lung transplantation, and a therapeutic approach of cyclical rituximab infusions has been associated with stability of his very severe CTD associated ILD over the last 12 months.

\section{Patient 7}

This 37-yr-old lady presented with widespread consolidation on HRCT and was managed initially as non-resolving pneumonia. Worsening respiratory failure resulted in the initiation of mechanical ventilation and treatment with i.v. methylprednisolone (one gram daily for 3 days; without clinical improvement), and the patient was transferred to our ITU. Positive autoimmune serology (rheumatoid factor 1:1,720, anti-Ro antibodies), HRCT appearances of organising pneumonia with diffuse alveolar damage, and negative microbiology (including virology) on bronchoalveolar lavage (BAL), resulted in the decision to treat with rituximab and reduced dose i.v. cyclophosphamide (750 mg).

Following rituximab, an episode of suspected CMV pneumonitis was successfully treated with aciclovir (more details can be found in the online supplementary material), and HRCT appearances improved significantly in the subsequent 4 weeks. The patient was discharged home after 9 weeks. 8 months following the initial rituximab dose, widespread consolidation re-appeared on HRCT on a background of fibrotic changes and, following BAL to exclude infection, a second cycle of rituximab was administered. Dyspnoea gradually improved over the subsequent 3 months, although significant residual disability remains.

\section{SSc}

Patient 8

This 63-yr-old male had longstanding SSc-associated NSIP, experienced deteriorating symptoms and HRCT imaging despite mycophenylate and prednisolone. Significant haematuria with cyclophosphamide precluded the further use of this agent. Although there was symptomatic improvement and improved in 6-min walk distance following rituximab (from 198 to $264 \mathrm{~m}$ ), there was no significant change in PFTs at 9 months follow-up.

\section{DISCUSSION}

The observation that rituximab is an effective rescue therapy in some patients with severe CTD-ILD has important clinical implications. If these findings are validated by further experience, it can no longer be assumed that patients with severe progressive CTD-ILD have the same bleak treated outcome as those with end-stage fibrotic idiopathic interstitial pneumonia. We report objective improvement in seven of eight patients (with very striking improvements in five patients) in whom the only realistic outcomes appeared to be lung transplantation or early death.

Although this study can be criticised as observational, it appears highly unlikely that a placebo-controlled evaluation 
could be performed in this context. In the first instance, patients and many clinicians will find a placebo-controlled trial unacceptable in the setting of life-threatening disease for which there is open therapy available with a good conceptual basis for its use. The selective bias against patients with more severe disease, when open treatment is accessible, has been highlighted as one of the key obstacles to observing clear-cut benefits in placebo-controlled trials in SSc-ILD [22], and in chronic pulmonary sarcoidosis [23]. Secondly, careful clinical observational studies based on treatment by proof of concept, rather than evidence base, may be the only feasible option in rare and/or clinically heterogeneous ILDs as those described here. Finally, severe end-stage disease is less attractive to pharmaceutical and other funding bodies, in view of a greater risk of non-efficacy and applicability only to a small patient subset, as highlighted by the exclusion of patients with more severe disease in most of the large recently completed clinical trials in IPF [24-29].

While our report is observational (with the inherent limitations), our analysis was a cross-over design, and thus the treatment effect was intrinsically more convincing than an evaluation of de novo therapy with no reference to previous lung function trends. Two series have previously reported the efficacy of rituximab in CTD-ILD [18, 19]. Importantly, both series included patients with much less severe interstitial lung involvement than our own cohort. SEM et al. [18] reported a positive effect of rituximab in seven of 11 patients with antisynthetase syndrome-associated ILD, the majority of whom had failed conventional immunosuppression [18]. Although complete lung function data is not reported, graphical representation of available baseline measurements suggests that DL,CO and FVC were only mildly to moderately impaired ( $\mathrm{LL}, \mathrm{CO} \geqslant 60 \%$ predicted in eight of nine patients, FVC $\geqslant 50 \%$ in six patients), in contrast with the markedly more severe lung disease in our PM/DM cohort (table 2). In SSc-associated ILD, DAOUsSis et al. [19] and colleagues reported a significant improvement in PFTs following rituximab treatment (compared to standard therapy). Again, rituximab-treated patients in this cohort had less severe ILD (mean \% predicted DL,CO of $52 \pm 21 \%$ and FVC of $68 \pm 20 \%$ ).

While little prognostic data exists for very severe and progressive CTD-ILD, clinical experience suggests an expected survival of 6-12 months in patients with similar disease severity as those described in our cohort. Our results suggest that, in the context of underlying autoimmunity, even patients with very severe ILD (for whom we would usually plan palliative treatment) may be rescued with rituximab, despite disease that is unresponsive to all traditional treatments. Immunological over-activity appears to be a key process in progressive lung fibrosis, even when fibrosis is severe, and the effectiveness of rituximab suggests that B lymphocytes are key protagonists in disease progression.

Several important caveats should be highlighted in this report. Life-threatening disease severity in patients 1 and 7 (both of whom were mechanically ventilated at the time of referral), resulted in the decision to administer rituximab and i.v. cyclophosphamide concurrently. This does make an accurate assessment of the stand-alone effects of rituximab difficult to ascertain. However, cyclophosphamide was administered only twice, at a reduced dose (50-75\% of the dose routinely used), whereas the standard regimen for ILD-CTD would have included six monthly doses (at $600 \mathrm{mg} \cdot \mathrm{m}^{-2}$ each) [30]. It therefore seems unlikely that the small dose of cyclophosphamide was effective on its own in controlling devastating ILD, although an additive effect cannot be excluded. In the other patients, the effect of concomitant or preceding immunosuppression needs to be considered as a potential confounding factor. Three received treatment with i.v. cyclophosphamide before switching to rituximab. Although a delayed effect of i.v. cyclophosphamide cannot be excluded with absolute certainty, stabilisation and/or improvement in lung function was observed in responders within 6 months of initiation of cyclophosphamide in a large retrospective cohort of patients with progressive fibrotic lung disease [31]. The remaining three patients had continued to progress while on mycophenolate, and response to rituximab seems the most plausible explanation for the observed improvement.

A negligible treatment effect was observed in one patient (patient 8) following rituximab. As described, this 60-yr-old male had SSc-associated fibrotic NSIP for $>10$ years and, while there was a definite improvement in symptoms and exercise capacity at 9 months, there was no significant change in PFTs.

Increased risk of infection following rituximab is a serious concern. In haematological malignancies, repeated doses of rituximab appear to be associated with an increased infection risk $[32,33]$, although this is not clearly apparent in rheumatological disease. In a recent meta-analysis of 745 patients with rheumatoid arthritis (RA), the rate of serious infections was not significantly different between rituximab and placebo-treated patients $(2.3 \%$ versus $1.5 \%)$ [34]. In a long-term safety analysis of 2,578 patients with RA treated with at least one course of rituximab, the rate of severe infections did not increase with subsequent doses, despite an increased incidence of hypogammaglobulinaemia [35]. Progressive multifocal leukoencephalopathy has been described in rituximab-treated patients. Although the reported incidence is extremely low (less than $1: 20,000$ in rituximab-treated RA patients) [36], patients should be monitored closely for the development of new or worsening neurological symptoms. Rituximab induced pulmonary reactions have also been reported, with steroid responsive organising pneumonia the most common manifestation in one systematic review [37].

Two patients in our cohort had significant infectious complications following rituximab. Patient 7 experienced recurrent lower respiratory tract infections following rituximab and patient 5 died of a respiratory tract infection 18 months after rituximab. Patient 5 had commenced cyclosporine (in addition to longstanding mycophenolate) to better control his myositis, 4 months prior to the fatal respiratory infection, and the contribution of rituximab in predisposing to infection is difficult to establish. Neither patient experienced reduced immunoglobulin levels during follow-up. The possibility that rituximab-induced serious lung infections are more frequent in patients with ILD compared to autoimmune diseases without significant lung involvement will need to be evaluated further. 


\section{Conclusion}

In a selected group of patients with severe, progressive CTDassociated ILD resistant to conventional immunosuppressive treatment, rituximab therapy was associated with significant clinical and functional improvements. Further work is needed to confirm these findings, delineate the pathways through which B cell ablation may inhibit lung fibrosis and to evaluate best re-treatment regimens and assess longer term outcomes and safety concerns.

\section{STATEMENT OF INTEREST}

Statements of interest for T.M. Maher and C. P. Denton can be found at www.erj.ersjournals.com/site/misc/statements.xhtml

\section{REFERENCES}

1 Latsi PI, du Bois RM, Nicholson AG, et al. Fibrotic idiopathic interstitial pneumonia: the prognostic value of longitudinal functional trends. Am J Respir Crit Care Med 2003; 168 : 531-537.

2 Luzina IG, Atamas SP, Wise R, et al. Gene expression in bronchoalveolar lavage cells from scleroderma patients. Am J Respir Cell Mol Biol 2002; 26: 549-557.

3 Lafyatis R, O'Hara C, Feghali-Bostwick CA, et al. B cell infiltration in systemic sclerosis-associated interstitial lung disease. Arthritis Rheum 2007; 56: 3167-3168.

4 Bosello S, De Santis M, Lama G, et al. B cell depletion in diffuse progressive systemic sclerosis: safety, skin score modification and IL-6 modulation in an up to thirty-six months follow-up openlabel trial. Arthritis Res Ther 2010; 12: R54.

5 Chino $\mathrm{Y}$, Murata $\mathrm{H}$, Goto $\mathrm{D}$, et al. $\mathrm{T}$ cell receptor BV gene repertoire of lymphocytes in bronchoalveolar fluid in polymyositis/dermatomyostis patients with interstitial pneumonitis. Int J Mol Med 2006; 17: 101-109.

6 Stone KB, Oddis CV, Fertig N, et al. Anti-Jo-1 antibody levels correlate with disease activity in idiopathic inflammatory myopathy. Arthritis Rheum 2007; 56: 3125-3131.

7 Yamadori I, Fujita J, Kajitani H, et al. Lymphocyte subsets in lung tissues of non-specific interstitial pneumonia and pulmonary fibrosis associated with collagen vascular disorders: correlation with CD4/CD8 ratio in bronchoalveolar lavage. Lung 2000; 178: 361-370.

8 D'Cruz D, Kamastha M, Hughes G. Pulmonary manifestations of systemic lupus erythematosis. In: Wallace DJ, Hahn B, Dubois D, eds. Dubois' lupus erythematosis. 6th Edn. Philidelphia, Lippincott Williams and Wilkins; 2002. pp. 666-683.

9 Perosa F, Prete M, Racanelli V, et al. CD-20 depleting therapy in autoimmune disease: from basic research to the clinic. J Intern Med 2010; 267: 260-277.

10 Leandro MJ, Cambridge G, Ehrenstein MR, et al. Reconstitution of peripheral blood $\mathrm{B}$ cells after depletion with rituximab in patients with rheumatoid arthritis. Arthritis Rheum 2006; 54 $613-620$

11 Edwards JC, Szczepanski L, Szechinski J, et al. B-cell-targeted therapy with rituximab in patients with rheumatoid arthritis. N Engl J Med 2004; 350: 2572-2581.

12 Emery P, Fleischmann R, Filipowicz-Sosnowska A, et al. (DANCER Study Group).The efficacy and safety of rituximab in patients with active rheumatoid arthritis despite methotrexate treatment: results of a phase IIB randomized, double-blind, placebo-controlled, dose-ranging trial. Arthritis Rheum 2006; 54 1390-1400.

13 Cohen SB, Emery P, Greenwald MW, et al. (REFLEX Trial Group). Rituximab for rheumatoid arthritis refractory to anti-tumor necrosis factor therapy: results of a multicenter, randomized, doubleblind, placebo-controlled, phase III trial evaluating primary efficacy and safety at twenty-four weeks. Arthritis Rheum 2006; 54: 2793-2806.

14 Jones RB, Tervaert JW, Hauser $\mathrm{T}$, et al. Rituximab versus cyclophosphamide in ANCA-associated renal vasculitis. $N$ Engl J Med 2010; 363: 211-220.

15 Stone JH, Merkel PA, Spiera R, et al. Rituximab versus cyclophosphamide for ANCA-associated vasculitis. N Engl J Med 2010; 363: 221-232.

16 Kavuru MS, Malur A, Marshall I, et al. An open-label trial of rituximab therapy in pulmonary alveolar proteinosis. Eur Respir $J$ 2011; 38: 1361-1367.

17 Arnold DM, Dentali F, Crowther MA, et al. Systematic review: efficacy and safety of rituximab for adults with idiopathic thrombocytopenic purpura. Ann Int Med 2007; 146: 25-44.

18 Sem M, Molberg O, Lund MB, et al. Rituximab treatment of the antisynthetase syndrome: a retrospective case series. Rheumatology (Oxford) 2009; 48: 968-971.

19 Daoussis D, Liossis SN, Tsamandas AC, et al. Experience with rituximab in scleroderma: results from a 1-year, proof-of-principle study. Rheumatology (Oxford) 2010; 49: 271-280.

20 Daoussis D, Liossis SN, Tsamandas AC, et al. Is there a role for B-cell depletion as therapy for scleroderma? A case report and review of the literature. Semin Arthritis Rheum 2010; 40: 127-136.

21 Vandenbroucke E, Grutters JC, Altenburg J, et al. Rituximab in life threatening antisynthetase syndrome. Rheumatol Int 2009; 29: 1499-1502.

22 Wells AU, Latsi P, McCune WJ. Daily cyclophosphamide for scleroderma: are patients with the most to gain underrepresented in this trial? Am J Respir Crit Care Med 2007; 176: 952-953.

23 Wells AU, Hogaboam CM. Update in diffuse parenchymal lung disease 2006. Am J Respir Crit Care Med 2007; 175: 655-660.

24 King TE Jr, Albera C, Bradford WZ, et al. Effect of interferon gamma-1b on survival in patients with idiopathic pulmonary fibrosis (INSPIRE): a multicentre, randomised, placebo-controlled trial. Lancet 2009; 374: 222-228.

25 Noble PW, Albera C, Bradford WZ, et al. Pirfenidone in patients with idiopathic pulmonary fibrosis (CAPACITY): two randomised trials. Lancet 2011; 377: 1760-1769.

26 King TE Jr, Behr J, Brown KK, et al. BUILD-1: a randomized placebo-controlled trial of bosentan in idiopathic pulmonary fibrosis. Am J Respir Crit Care Med 2008; 177: 75-81.

27 King TE Jr, Brown KK, Raghu G, et al. BUILD-3: a randomized, controlled trial of bosentan in idiopathic pulmonary fibrosis. Am J Respir Crit Care Med 2011; 184: 92-99.

28 Raghu G, Brown KK, Costabel U, et al. Treatment of idiopathic pulmonary fibrosis with etanercept: an exploratory, placebo-controlled trial. Am J Respir Crit Care Med 2008; 178: 948-955.

29 Daniels CE, Lasky JA, Limper AH, et al. Imatinib treatment for idiopathic pulmonary fibrosis: Randomized placebocontrolled trial results. Am J Respir Crit Care Med 2010; 181: 604-610.

30 Hoyles RK, Ellis RW, Wellsbury J, et al. A multicenter, prospective, randomized, double-blind, placebo-controlled trial of corticosteroids and intravenous cyclophosphamide followed by oral azathioprine for the treatment of pulmonary fibrosis in scleroderma. Arthritis Rheum 2006; 54: 3962-3970.

31 Corte TJ, Ellis R, Renzoni EA, et al. Use of intravenous cyclophosphamide in known or suspected, advanced nonspecific interstitial pneumonia. Sarcoidosis Vasc Diffuse Lung Dis 2009; 26: 132-138.

32 Aksoy S, Dizdar O, Hayran M, et al. Infectious complications of rituximab in patients with lymphoma during maintenance therapy: 
a systematic review and meta-analysis. Leuk Lymphoma 2009; 50: 357-365.

33 Vidal L, Gafter-Gvili A, Leibovici L, et al. Rituximab as maintenance therapy for patients with follicular lymphoma. Cochrane Database Syst Rev 2009; 2: CD006552.

34 Salliot C, Dougados M, Gossec L. Risk of serious infections during rituximab, abatacept and anakinra treatments for rheumatoid arthritis: meta-analyses of randomised placebo-controlled trials. Ann Rheum Dis 2009; 68: 25-32.
35 van Vollenhoven RF, Emery P, Bingham CO 3rd, et al. Longterm safety of patients receiving rituximab in rheumatoid arthritis clinical trials. J Rheumatol 2010; 37: 558-567.

36 Buch $\mathrm{MH}$, Smolen JS, Betteridge N, et al. Updated consensus statement on the use of rituximab in patients with rheumatoid arthritis. Ann Rheum Dis 2011; 70: 909-920.

37 Lioté H, Lioté F, Séroussi B, et al. Rituximab-induced lung disease: A systematic literature review. Eur Respir J 2010; 35: 681-687. 